Canadian

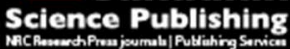

Applied Physiology, Nutrition, and Metabolism Physiologie appliquée, nutrition et métabolisme

\title{
Investigating human skeletal muscle physiology with unilateral exercise models: when one limb is more powerful than two
}

\begin{tabular}{|r|l|}
\hline Journal: & Applied Physiology, Nutrition, and Metabolism \\
\hline Manuscript ID & apnm-2016-0645.R1 \\
\hline Manuscript Type: & Review \\
\hline Date Submitted by the Author: & 22-Dec-2016 \\
\hline Complete List of Authors: & $\begin{array}{l}\text { MacInnis, Martin; McMaster University, Kinesiology } \\
\text { McGlory, Chris; McMaster University, Kinesiology } \\
\text { Gibala, Martin; McMaster University, } \\
\text { Phillips, Stuart; McMaster University, }\end{array}$ \\
\hline Keyword: & $\begin{array}{l}\text { Experimental Design, nutrition < nutrition, Skeletal muscle biopsy, Aerobic } \\
\text { training, resistance training < exercise }\end{array}$ \\
\hline
\end{tabular}


Investigating human skeletal muscle physiology with unilateral exercise models: when one limb is more powerful than two

Martin J. MacInnis ${ }^{1}$, Chris McGlory ${ }^{1}$, Martin J. Gibala ${ }^{1}$, Stuart M. Phillips ${ }^{1}$

${ }^{1}$ Department of Kinesiology, McMaster University, Hamilton, Ontario, Canada

\section{Corresponding Author:}

Stuart M. Phillips, PhD

Professor

Department of Kinesiology

McMaster University

1280 Main Street West

Hamilton, ON

L8S-4K1

Canada

Email: phillis@mcmaster.ca

Telephone: +1 9055259140 (ext. 24465)

\section{Additional Email Addresses:}

MJM: macinnm@mcmaster.ca

CM: mcglorc@mcmaster.ca

MJG: gibalam@mcmaster.ca

WORD COUNT: 7096 (including references and figure legends) 


\begin{abstract}
Direct sampling of human skeletal muscle using the needle biopsy technique can facilitate insight into the biochemical and histological responses resulting from changes in exercise or feeding. However, the muscle biopsy procedure is invasive, and analyses are often expensive, which places pragmatic restraints on sample sizes. The unilateral exercise model can serve to increase statistical power and reduce the time and cost of a study. With this approach, two limbs of a participant are randomized to one of two treatments that can be applied almost concurrently or sequentially depending on the nature of the intervention. Similar to a typical repeated measures design, comparisons are made within participants, which increases statistical power by reducing the amount of between-person variability. A washout period is often unnecessary, reducing the time needed to complete the experiment and the influence of potential confounding variables such as habitual diet, activity, and sleep. Variations of the unilateral exercise model have been employed to investigate the influence of exercise, diet, and the interaction between the two, on a wide range of variables including mitochondrial content, capillary density and skeletal muscle hypertrophy. Like any model, unilateral exercise has some limitations: it cannot be used to study variables that potentially transfer across limbs, and it is generally limited to exercises that can be performed in pairs of treatments. Where appropriate, however, the unilateral exercise model can yield robust, well-controlled investigations of skeletal muscle responses to a wide range of interventions and conditions including exercise, dietary manipulation, and disuse or immobilization.
\end{abstract}

\title{
Abstract word count: 249
}

Keywords: Nutrition, experimental design, skeletal muscle biopsy, aerobic training, resistance training 


\section{Introduction}

Our understanding of how exercise influences skeletal muscle remodelling at the cellular and molecular levels is largely due to the development of the skeletal muscle biopsy technique, a method that has been in practice for more than 60 years (Bergstrom 1962, Bergström and Hultman 1967, Bergström et al. 1967). Due in part to the financial cost of muscle sample analyses, ethical considerations that limit the number of biopsies from participants, and the need for skilled practitioners to collect the muscle samples, studies employing this invasive procedure often involve small sample sizes designed to detect minimal significant differences. This restriction in sample size often presents a challenge from a research design standpoint since statistical power is partly dependent on the number of participants included in the study. While the sample size cannot always be increased, manipulating the experimental design provides an alternative means to increase the statistical power of an experiment. Crossover designs are often desirable because they provide greater statistical power compared to parallel group designs (Figures 1 and 2). This is true even when confounding factors are well controlled in parallel group designs. The use of crossover designs to reduce inter-subject variation in human research studies is particularly attractive when primary outcomes are regulated at the transcriptional and translational levels.

One particular application of the crossover design, which can be employed in human skeletal muscle biopsy studies, involves the unilateral exercise model (Figure 1). This design typically entails the randomization of the participant's limbs (arms or legs) to different treatments that are often administered in close temporal succession (i.e., almost concurrently), with comparisons made between limbs but within a participant. Under certain conditions, the unilateral exercise model affords a strong design to compare exercise protocols (e.g., endurance 
vs. resistance exercise), variations of a similar protocol (e.g., low- vs. high-load resistance exercise), and various stimuli in reference to a control (e.g., unilateral limb immobilization). The primary purpose of this brief review is to discuss the assumptions, advantages, disadvantages, and applications of the unilateral exercise model in experimental research investigating skeletal muscle adaptive responses to exercise in humans.

\section{Assumptions of the unilateral exercise model}

The main assumptions of the unilateral exercise model are that the two limbs of an individual are equally responsive to the potential treatments and that each limb is unaffected by the treatment in the contralateral limb. As long as the limbs of an individual are reasonably well matched at baseline, their responsiveness to a change in contractile pattern or feeding should theoretically be similar. In contrast, the potential for a training response to 'transfer' from one limb to another (e.g., from the training limb to the control limb) seems to depend on the nature of the outcome being investigated.

Comparisons that are made within participants, using the unilateral exercise model, greatly reduce the potential influence of inter-individual differences on the results obtained. Yet, it is possible that baseline differences could influence the magnitude of the responses from each limb. This scenario is unlikely, however, as skeletal muscle biochemical and histological characteristics appear to be similar between limbs, albeit with most data generated from studies of the quadriceps. For example, whole muscle volume (Tesch et al. 2004, Suetta et al. 2009) and cross sectional area (Wilkinson et al. 2006, West et al. 2010), fibre type cross-sectional area (Saltin et al. 1976, Henriksson 1977, Essen-Gustavsson and Borges 1986, Lexell and Taylor 1991, Verdijk et al. 2007, Tarnopolsky et al. 2007), mitochondrial content, measured via enzyme 
activities and mitochondrial respiration (Saltin et al. 1976, Essen-Gustavsson and Borges 1986, Hansen et al. 2005, MacInnis et al. 2016, Lindholm et al. 2016), capillary:fibre ratio (Jensen et al. 2004), glycogen content (Hansen et al. 2005), blood lactate production (Saltin et al. 1976, Henriksson 1977), mRNA abundance (Tarnopolsky et al. 2007, Lindholm et al. 2014), and signalling protein phosphorylation (Coffey et al. 2010) are similar between legs of an individual at baseline. That variation in resting gene expression was nearly five times greater between individuals than it was between the legs of an individual (Lindholm et al. 2014) underscores the potential for the unilateral exercise model to reduce variability. Aerobic capacity (Saltin et al. 1976, Henriksson 1977, MacInnis et al. 2016) and one-repetition maximum for leg extension (Secher et al. 1988, Schantz et al. 1989) are also similar between legs. Finally, there is little difference in muscle protein turnover rates between different muscle groups in humans despite quite marked differences in fibre types (Mittendorfer et al. 2005).

Differences in muscular power and strength between left and right legs are seemingly small, but these differences are more pronounced when legs are grouped according to dominance (Newton et al. 2006). Concerns in this regard are mitigated through the random assignment of limbs, based on dominance, to the two treatment groups. While self-report is likely sufficient for determining handedness, a simple to administer questionnaire (e.g., the Waterloo Footedness Questionnaire - Revised) can help determine a participant's dominant leg (Elias et al. 1998). A common criticism of unilateral exercise training is that skeletal muscle adaptations in one limb can transfer to the contralateral limb, invalidating this model. For instance, resistance exercise is known to result in a transient increase in a variety of systemic and purportedly anabolic hormones (i.e., growth hormone, insulin-like growth factor, testosterone) that enter the circulation following exercise (West and Phillips 2012). The release of these hormones into the 
circulatory system in response to exercise could therefore confound the physiological response of the contralateral limb. This supposition has been challenged, as many studies have shown that the acute systemic elevations in such hormones play little-to-no role in exercise-induced increases in either muscle protein synthesis or skeletal muscle cross sectional area [reviewed elsewhere (West and Phillips 2010)]. Similarly, molecules released from skeletal muscle during unilateral exercise (e.g., 'myokines' and 'exerkines') could theoretically influence the contralateral limb (Safdar et al. 2016, Whitham and Febbraio 2016); nevertheless, these effects would most likely be small compared to the direct effects of exercise on the muscle.

Biochemical responses to exercise (e.g., protein and/or mRNA abundance) show no measurable signs of transfer between limbs, whereas neuromuscular responses to exercise seemingly do (e.g., motor unit recruitment). An apparent lack of transfer of skeletal muscle adaptations from the exercising limb to the non-exercising limb has been demonstrated for citrate synthase (i.e., mitochondrial content) (Bell et al. 2001, Lundberg et al. 2013, Lindholm et al. 2016), 3-hydroxyacyl-CoA dehydrogenase (Kiens et al. 1993, Rud et al. 2012, Lindholm et al. 2016), and succinate dehydrogenase maximal activities (Saltin et al. 1976, Henriksson 1977, Kiens et al. 1993), muscle capillarization (Jensen et al. 2004), local hemodynamics (Kiens et al. 1993, Mourtzakis et al. 2004, Rud et al. 2012), metabolic adaptations during submaximal exercise (e.g., decreased lactate concentrations and glycogenolysis rates and increased free fatty acid uptake) (Saltin et al. 1976, Henriksson 1977, Kiens et al. 1993), as well as skeletal muscle protein synthesis (Miller et al. 2005) and hypertrophy (Houston et al. 1983, Wilkinson et al. 2006). In contrast, the cross-education effect (i.e., increased strength in the untrained arm following unilateral resistance training) has been demonstrated numerous times (Carroll et al. 2006), although it is likely due to neuromuscular, not biochemical, adaptations (Lee and Carroll 
2007). The influence of the cross-education effect may be minimal when both limbs are being trained within a given intervention (Munn 2004) or the non-dominant arm is being trained (Farthing et al. 2005).

\section{Advantages of the unilateral exercise model}

The unilateral exercise model and crossover design share the same primary advantage compared to parallel group designs: the lack of inter-subject variability reduces the required sample size to obtain adequate statistical power in outcomes of interest (Figure 2). As the unilateral exercise model allows two treatments to be administered almost concurrently, this model provides additional advantages relative to the crossover design. For example, there is no need for a washout period, there is greater control of between-subject confounding factors, and there is often a reduced cost and participant burden.

Washout periods lasting weeks to months are often needed to allow skeletal muscle adaptations to abate after chronic training (Henriksson and Reitman 1977, Klausen et al. 1981, Mujika and Padilla 2000). The unilateral exercise model usually does not require a washout period between treatments, because exercise-specific adaptations generally do not cross over to the untrained limb, enabling treatments to be delivered almost concurrently or at least temporally close to each other. Eliminating washout periods greatly reduces the amount of time needed to perform a study, which likely decreases the normal variability that occurs over time and the likelihood of participant attrition.

For exercise interventions, crossover designs are generally preferable to parallel-group designs from a statistical power perspective because within-participant comparisons reduce the influence of between-participant variability (Bouchard et al. 1999, Churchward-Venne et al. 
2015), which likely has some genetic influence (Bouchard et al. 1999). However, further advantages can be gained with the unilateral exercise model because responses to exercise may also be affected by factors such as nutrition, fitness, and sleep (Hawley et al. 2011, Halson 2014). The nearly concurrent application of treatments would reduce variability stemming from changes in these extraneous factors that could occur during the time between treatments in a crossover design (i.e., during a washout period). Thus, with the unilateral exercise model, greater statistical power would be expected relative to crossover designs because of the reduced influence of confounding factors. Regardless of the experimental design, unilateral training provides a more homogenous aerobic training stimulus than bilateral training: the fraction of each leg's aerobic capacity that is utilized is highly variable during two-leg cycling (i.e., ranging from 58-96\%), but it can be controlled during single-leg cycling (McPhee et al. 2009).

A major consideration in studies of skeletal muscle physiology is controlling for differences in nutritional status (Hawley et al. 2011). One of the primary strengths of the unilateral model is that it enables comparisons of different treatments under identical nutritional states. Specifically, if trained on the same day, the pre-exercise nutrition state is highly similar for the two limbs, and the post-exercise provision of nutritional stimuli enters the circulatory system and reaches both limbs simultaneously. For chronic studies, alternating the order of training sessions across days should minimize any effects of delaying nutrient delivery in one limb (Morton et al. 2015). From a practical perspective, the unilateral exercise model can reduce experimental costs associated with an experiment. Providing food to participants to control diet, especially for extended periods of time, is one or the largest expenses of human trials. With the unilateral exercise model, the reduction in participants and the overlap of the treatments greatly reduces this cost, as well as the effort to prepare and deliver food. Similarly, for an acute trial 
measuring muscle protein synthesis with stable isotope tracers, half the amount of tracer is necessary for unilateral exercise trials compared to crossover designs, providing a significant cost advantage. The decreased number of participants required for unilateral exercise studies generates fewer muscle biopsy samples, reducing the cost and effort of data collection and analysis.

Although it is not an advantage per se, it is important to note that unilateral resistance and aerobic exercise are quite easy, particularly with familiarization, to perform. Resistance exercise training normally consists of both bilateral and unilateral modes. Accordingly, unilateral resistance exercise can be performed using unmodified commercially available equipment (Mitchell et al. 2012, McGlory et al. 2016), and minimal training is likely be needed to familiarize most participants with the prescribed unilateral manoeuvres (e.g., single-leg knee extensions or leg press, or single-arm biceps curls). In contrast, aerobic exercise is typically performed bilaterally (e.g., running, cycling, swimming, rowing); therefore, unilateral aerobic exercise can require specialized laboratory equipment and more familiarization than the bilateral alternative. The most common modes of unilateral aerobic exercise are dynamic knee extensions (Andersen et al. 1985), which require a specialized device, and single-leg cycling (Saltin et al. 1976, Rud et al. 2012), which does not necessarily require a modified cycle ergometer (Dolmage et al. 2014) but requires familiarization. Our research groups have frequently used unilateral modes of exercise to study acute responses (West et al. 2009, Burd et al. 2010, 2012, Churchward-Venne et al. 2012) and chronic adaptations (Wilkinson et al. 2006, West et al. 2010, Mitchell et al. 2012, MacInnis et al. 2016) to aerobic and resistance exercise.

Although the focus of this review is the utility of the unilateral exercise model for comparing different exercise conditions, unilateral exercise was instrumental for studying factors 
limiting $\mathrm{VO}_{2} \max$ (Davies and Sargeant 1974, Saltin et al. 1976). Specifically, using the dynamic knee extension model (Andersen et al. 1985), Andersen and Saltin (1985) demonstrated that skeletal muscle blood flow was greater for unilateral than bilateral aerobic exercise, indicating that the capacity of skeletal muscle to accommodate blood flow exceeded the capacity of the circulatory system to supply it.

\section{Disadvantages and limitations to unilateral exercise models}

There are some disadvantages and limitations with the unilateral exercise model. The more apparent limitations include restrictions in the types of exercise, number of treatments, and range of variables that can be studied with unilateral exercise. Whether or not unilateral and bilateral aerobic and resistance exercise elicit comparable responses requires more research.

Not all muscle groups are ideal for comparisons of resistance training protocols and whole-body unilateral training protocols may not be feasible for some experiments. For example, it is possible to train muscles in the back, shoulders, and chest unilaterally (Chilibeck et al. 2004, Janzen et al. 2006), but biopsy samples are not routinely collected from these sites, limiting their inclusion in biochemical analysis. Similarly, it is possible to perform unilateral exercise with the major muscle groups for one side of the body independent of the contralateral side (Chilibeck et al. 2004); however, it may be unwise to involve so many muscles (i.e., 'whole-body' training) when comparing protocols expected to have very different results, such as exercise vs. a nonexercise control, as a potentially deleterious asymmetry could develop. Accordingly, unilateral exercise studies are focused on the musculature of the limbs, which are easier to sample, and this sampling preference could have implications for generalizability (Macnaughton et al. 2016). For aerobic exercise, cycling and knee extensions are the predominant modes of unilateral exercise 
performed in most studies. Thus, the generalizability of data obtained from unilateral knee extensions and cycling is uncertain for other forms of aerobic exercise, such as running.

An obvious limitation to this model is that only two treatments can be compared simultaneously. While many researchers might be interested in comparing more than two treatments, the increased statistical power and the elimination of washout periods could reduce the required sample size and study duration enough to permit multiple studies for a given budget and time allotment. Such a design would still have some statistical superiority over a complete between-subject design since some measures would be within subjects. This does require a nested statistical analysis, however, and recognition that between-subject variation is likely to be greater than within-subject variation. Thus, researchers would have to be careful to pair the protocols such that the most important comparisons are performed within the same individuals.

The use of a unilateral exercise model is limited to those variables that do not transfer from the exercised limb to the contralateral limb. Traits such as muscular strength are not well suited for unilateral exercise training due to the cross-education phenomenon as discussed above. Additionally, systemic responses to exercise training stimuli cannot be studied with unilateral exercise. For example, unilateral aerobic exercise training is not suitable for comparing the effects of various exercise stimuli on central adaptations (e.g., cardiac output and aerobic capacity) or whole-body glucose homeostasis, variables that are often studied in combination with aspects of skeletal muscle physiology (Gillen et al. 2016, Bækkerud et al. 2016).

Due to bilateral deficits, responses elicited by unilateral and bilateral aerobic and resistance exercise may not necessarily be of the same magnitudes. Central limitations to bilateral aerobic exercise permit greater workloads per leg for unilateral as compared to bilateral cycling (Pernow and Saltin 1971, Klausen et al. 1982), potentially providing a greater stimulus 
for the muscles when legs are trained separately. For example, when cyclists performed highintensity interval training with one or two legs at the same relative intensity (i.e., maximal maintainable effort for 5-min), skeletal muscle adaptations were greater for single-legged cycling (Abbiss et al. 2011). Similarly, single-leg cycling was more effective than double-leg cycling for increasing aerobic capacity and peak power output in individuals with chronic obstructive pulmonary disease undergoing supervised training (Dolmage 2008). A bilateral deficit in force production is also apparent with resistance exercise models (Škarabot et al. 2016); however, increases in strength, power, and lean body mass following short-term resistance exercise training appear comparable for unilateral and bilateral exercise (McCurdy et al. 2005, Janzen et al. 2006, Costa et al. 2015). More research is needed to understand whether unilateral and bilateral exercise elicit the same acute molecular and metabolic responses when performed at the same absolute and relative intensities. Potential differences in the response to unilateral and bilateral exercise do not invalidate the model: similar adaptations would be expected for unilateral and bilateral exercise when equal work was performed in the same pattern (if possible), and relative differences between unilateral protocols would still likely apply to bilateral exercise.

\section{Applications of unilateral exercise training}

In our opinions, the use of unilateral exercise as the training intervention is often advantageous to bilateral exercise because it allows for more efficient experiments (e.g., reduced participants, time, and cost), which is critical for studies collecting skeletal muscle biopsy samples from humans. Thus, the unilateral exercise model has applications in a wide range of experiments, including those examining responses to different exercise protocols, the interaction of exercise and feeding, and the effects of disuse or immobilization. 
Unilateral exercise can be used to study basic physiological responses to aerobic and resistance exercise as well as the effects of dietary interventions on exercised/trained or rested/untrained muscle. For example, unilateral exercise is well suited to investigate skeletal muscle adaptations to different training programs [e.g., interval vs. continuous exercise (Saltin et al. 1976, MacInnis et al. 2016) and different physiological states [e.g., decreased muscle glycogen availability (Hansen et al. 2005) or increased circulating hormone concentrations (West et al. 2009, 2010)]. Furthermore, dietary stimuli can be delivered in combination with unilateral exercise to investigate their interactive effects. For example, to study how exercise modulates postprandial muscle protein turnover in response to resistance exercise, one limb can be trained and the contralateral limb can remain untrained, with protein provided post-training (Kim et al. 2005, Wilkinson et al. 2008, Churchward-Venne et al. 2012, McGlory et al. 2016). This approach has been employed by many researchers to yield informative data relating to how resistance exercise impacts responses to feeding, including the cellular and molecular processes of muscle growth (Mitchell et al. 2012, Witard et al. 2014, McGlory et al. 2016).

Theoretically, unilateral exercise could improve consistency between acute and chronic studies that compare the same treatments. In many instances, acute responses to two exercise treatments are compared prior to investigating their chronic effects, as has been performed frequently by our research groups when investigating the influence of circulating hormones (West et al. 2009, 2010), resistance exercise load (Mitchell et al. 2012, Morton et al. 2016), and carbohydrate availability (Cochran et al. 2010, 2015). The rationale behind these studies is that acute differences in the molecular response (e.g., gene expression, signalling pathway activation, protein synthesis) were expected to manifest as different training adaptations when performed repeatedly (e.g., protein content, enzyme activity, muscle mass). In many instances, acute trials 
utilize a crossover design because the washout period is relatively short for a single bout of exercise, whereas training studies have a parallel group design because the required washout period would be much too long to make many studies feasible. As discussed above, larger sample sizes are needed for parallel group designs to achieve the same power as crossover designs (Figure 2). As crossover designs are relatively difficult to implement for chronic training studies, unilateral exercise could be used for the training study to increase statistical power and provide a greater opportunity to recapitulate results from an acute trial.

Although the primary focus to this point has been on exercise training, a unilateral model of immobilization can also be used to elucidate factors underlying muscle atrophy and deconditioning (Tesch et al. 2004, Suetta et al. 2009, Vigelsø et al. 2015a, 2015b, Tesch et al. 2016). In these experiments, one limb is immobilized for a period of time using a cast (Farthing et al. 2009, Suetta et al. 2009) or brace (Glover et al. 2008, Vigelsø et al. 2015b) or one leg is unloaded through unilateral lower limb suspension (Tesch et al. 2004, Tesch et al. 2016, Lamboley et al. 2016, Perry et al. 2016). For instance, unilateral limb immobilization or unloading has shown that transient periods of disuse result in a profound decrease in muscle size, strength, and cross sectional area as well as the insidious onset of a host of metabolic risk factors (Tesch et al. 2004, Wall et al. 2013, Gram et al. 2014, Vigelsø et al. 2015b). Importantly, many of these adaptations are specific to the immobilized limb. For example, $14 \mathrm{~d}$ of single-leg bracing in young men results in a significant decline in isometric torque, thigh cross sectional area, and type I and II cross sectional area in the immobilized limb, with no detectable change in the contralateral limb (Figure. 3). Moreover, there were no detectable changes in the maximal activities of citrate synthase and beta-hydroxyacyl CoA dehydrogenase in the control leg (Figure 3). Similar results have been found by other groups (Tesch et al. 2004, Gram et al. 2014, Vigelsø 
et al. 2015b). Thus, as opposed to the often-cited criticism that in unilateral immobilization there is increased loading of the non-immobilized limb, we see no evidence of this effect at the phenotypic level. In fact, since a participant typically uses crutches with unilateral immobilization/suspension, the subject's body mass is supported across three, not two, points of contact and so is not a model of increased loading. It may be that unilateral immobilization is a model of global deconditioning. When participants were assessed before and after $12 \mathrm{~d}$ of immobilization, reductions in femoral and popliteal arterial compliance and femoral artery diameter were apparent in both the non-immobilized limb but to a much greater extent in the immobilized limb (Rakobowchuk et al. 2011). Given the lack of change in factors intrinsic to muscle in the non-immobilized control limb (e.g., muscle protein synthesis, cross-sectional area, enzymatic activities), it could be argued that such changes in arterial compliance are of little relevance when assessing histological or biochemical variables; however, whether this assertion would hold true during longer periods of immobilization remains unknown.

\section{Conclusions}

Unilateral modes of exercise permit within-participant comparisons of the biochemical and histological responses of skeletal muscle to nearly concurrent treatments. Both unilateral aerobic and resistance training are amenable to investigations of skeletal muscle remodelling in humans, and many important skeletal muscle adaptations occur without evidence of transfer to the contralateral limb. The unilateral exercise model provides greater control of extraneous variables and potentially greater statistical power compared to the typical crossover study, but it also reduces the amount of time, money, and labour needed to conduct an experiment. While there are important limitations to the use of the unilateral exercise model, including restrictions 
in the modes of exercise, number of simultaneous comparisons, and potential outcome variables, the unilateral exercise model is well suited for investigating the effects of exercise, nutrition, and unloading on human skeletal muscle.

\section{Conflict of interest}

The authors report no conflicts of interest associated with this manuscript.

\section{Acknowledgements}

The authors thank two annonymous reviewers for their considerate and condtructive suggestions, which helped improve the depth and quality of this review. 


\section{References}

Abbiss, C.R., Karagounis, L.G., Laursen, P.B., Peiffer, J.J., Martin, D.T., Hawley, J.A., Fatehee, N.N., and Martin, J.C. 2011. Single-leg cycle training is superior to double-leg cycling in improving the oxidative potential and metabolic profile of trained skeletal muscle. J. Appl. Physiol. 110(5): 1248-1255. doi:10.1152/japplphysiol.01247.2010.

Andersen, P., Adams, R.P., Sjøgaard, G., Thorboe, A., and Saltin, B. 1985. Dynamic knee extension as model for study of isolated exercising muscle in humans. J. Appl. Physiol. 59(5): 1647-1653.

Andersen, P., and Saltin, B. 1985. Maximal perfusion of skeletal muscle in man. J. Physiol. 366: 233-249.

Bell, C., Paterson, D.H., Kowalchuk, J.M., Moy, A.P., Thorp, D.B., Noble, E.G., Taylor, A.W., and Cunningham, D.A. 2001. Determinants of oxygen uptake kinetics in older humans following single-limb endurance exercise training. Exp. Physiol. 86(5): 659-665.

Bergstrom, J. 1962. Muscle electrolytes in man determined by neutron activation analysis on needle biopsy specimens. Scan. J. Clin. Lab. Invest. 14(Suppl 68). S: Teriks Sjukhus, Stockholm.

Bergström, J., and Hultman, E. 1967. A study of the glycogen metabolism during exercise in man. Scandinavian Scan. J. Clin. Lab. Invest. 19(3): 218-228. doi:10.3109/00365516709090629.

Bergström, J., Hermansen, L., Hultman, E., and Saltin, B. 1967. Diet, muscle glycogen and physical performance. Acta Physiol. Scand. 71(2): 140-150. doi:10.1111/j.17481716.1967.tb03720.x.

Bouchard, C., An, P., Rice, T., Skinner, J.S., Wilmore, J.H., Gagnon, J., Pérusse, L., Leon, A.S., and Rao, D.C. 1999. Familial aggregation of VO(2max) response to exercise training: results from the HERITAGE Family Study. J. Appl. Physiol. 87(3): 1003-1008.

Burd, N.A., Andrews, R.J., West, D.W.D., LITTLE, J.P., Cochran, A.J.R., Hector, A.J., Cashaback, J.G.A., Gibala, M.J., Potvin, J.R., Baker, S.K., and Phillips, S.M. 2012. Muscle time under tension during resistance exercise stimulates differential muscle protein subfractional synthetic responses in men. J. Physiol. 590(Pt 2): 351-362. doi:10.1113/jphysiol.2011.221200.

Burd, N.A., West, D.W.D., Staples, A.W., Atherton, P.J., Baker, J.M., Moore, D.R., Holwerda, 
A.M., Parise, G., Rennie, M.J., Baker, S.K., and Phillips, S.M. 2010. Low-load high volume resistance exercise stimulates muscle protein synthesis more than high-load low volume resistance exercise in young men. PLoS ONE, 5(8): e12033.

doi:10.1371/journal.pone.0012033.

Bækkerud, F.H., Solberg, F., Leinan, I.M., Wisløff, U., Karlsen, T., and Rognmo, Ø. 2016. Comparison of Three Popular Exercise Modalities on $\mathrm{V}^{\cdot} \mathrm{O} 2 \mathrm{max}$ in Overweight and Obese. Med Sci Sports Exerc 48(3): 491-498. doi:10.1249/MSS.0000000000000777.

Carroll, T.J., Herbert, R.D., Munn, J., Lee, M., and Gandevia, S.C. 2006. Contralateral effects of unilateral strength training: evidence and possible mechanisms. J. Appl. Physiol. 101(5): 1514-1522. doi:10.1152/japplphysiol.00531.2006.

Chilibeck, P.D., Stride, D., Farthing, J.P., and Burke, D.G. 2004. Effect of creatine ingestion after exercise on muscle thickness in males and females. Med. Sci. Sports Exerc. 36(10): 1781-1788.

Churchward-Venne, T.A., Burd, N.A., Mitchell, C.J., West, D.W.D., Philp, A., Marcotte, G.R., Baker, S.K., Baar, K., and Phillips, S.M. 2012. Supplementation of a suboptimal protein dose with leucine or essential amino acids: effects on myofibrillar protein synthesis at rest and following resistance exercise in men. J. Physiol. 590(11): 2751-2765. doi:10.1113/jphysiol.2012.228833.

Churchward-Venne, T.A., Tieland, M., Verdijk, L.B., Leenders, M., Dirks, M.L., de Groot, L.C.P.G.M., and van Loon, L.J.C. 2015. There Are No Nonresponders to Resistance-Type Exercise Training in Older Men and Women. J. Am. Med. Dir. Assoc. 16(5): 400-411. doi:10.1016/j.jamda.2015.01.071.

Cochran, A.J., Myslik, F., MacInnis, M.J., Percival, M.E., Bishop, D., Tarnopolsky, M.A., and Gibala, M.J. 2015. Manipulating Carbohydrate Availability Between Twice-Daily Sessions of High-intensity Interval Training Over Two Weeks Improves Time-trial Performance. Int. J. Sport Nutr. Exerc. Metab. doi:10.1123/ijsnem.2014-0263.

Cochran, A.J.R., Little, J.P., Tarnopolsky, M.A., and Gibala, M.J. 2010. Carbohydrate feeding during recovery alters the skeletal muscle metabolic response to repeated sessions of highintensity interval exercise in humans. J. Appl. Physiol. 108(3): 628-636. doi:10.1152/japplphysiol.00659.2009.

Coffey, V.G., Moore, D.R., Burd, N.A., Rerecich, T., Stellingwerff, T., Garnham, A.P., Phillips, 
S.M., and Hawley, J.A. 2010. Nutrient provision increases signalling and protein synthesis in human skeletal muscle after repeated sprints. Eur. J. Appl. Physiol. 111(7): 1473-1483. doi:10.1007/s00421-010-1768-0.

Costa, E., Moreira, A., Cavalcanti, B., Krinski, K., and Aoki, M. 2015. Effect of unilateral and bilateral resistance exercise on maximal voluntary strength, total volume of load lifted, and perceptual and metabolic responses. Biol. Sport. 32(1): 35-40. doi:10.5604/20831862.1126326.

Davies, C.T., and Sargeant, A.J. 1974. Physiological responses to one- and two-leg exercise breathing air and 45 percent oxygen. J. Appl. Physiol. 36(2): 142-148.

Dolmage, T.E. 2008. Effects of One-Legged Exercise Training of Patients With COPD* . Chest, 133(2): 370. doi:10.1378/chest.07-1423.

Dolmage, T.E., Evans, R.A., and Goldstein, R.S. 2014. A counterweight is not necessary to implement simple, natural and comfortable single-leg cycle training. Eur. J. Appl. Physiol. 114(11): 2455-2456. doi:10.1007/s00421-014-2948-0.

Elias, L.J., Bryden, M.P., and Bulman-Fleming, M.B. 1998. Footedness is a better predictor than is handedness of emotional lateralization. Neuropsychologia, 36(1): 37-43.

Essen-Gustavsson, B., and Borges, O. 1986. Histochemical and metabolic characteristics of human skeletal muscle in relation to age. Acta Physiol. Scand. 126(1): 107-114. doi:10.1111/j.1748-1716.1986.tb07793.x.

Farthing, J.P., Chilibeck, P.D., and Binsted, G. 2005. Cross-education of arm muscular strength is unidirectional in right-handed individuals. Med. Sci. Sports Exerc. 37(9): 1594-1600. doi:10.1249/01.mss.0000177588.74448.75.

Farthing, J.P., Krentz, J.R., and Magnus, C.R.A. 2009. Strength training the free limb attenuates strength loss during unilateral immobilization. J. Appl. Physiol. 106(3): 830-836. doi:10.1152/japplphysiol.91331.2008.

Gillen, J.B., Martin, B.J., MacInnis, M.J., Skelly, L.E., Tarnopolsky, M.A., and Gibala, M.J. 2016. Twelve Weeks of Sprint Interval Training Improves Indices of Cardiometabolic Health Similar to Traditional Endurance Training despite a Five-Fold Lower Exercise Volume and Time Commitment. PLoS ONE, 11(4): e0154075. doi:10.1371/journal.pone.0154075.

Glover, E.I., Phillips, S.M., Oates, B.R., Tang, J.E., Tarnopolsky, M.A., Selby, A., Smith, K., 
and Rennie, M.J. 2008. Immobilization induces anabolic resistance in human myofibrillar protein synthesis with low and high dose amino acid infusion. J. Physiol. 586(24): 60496061. doi:10.1113/jphysiol.2008.160333.

Gram, M., Vigelsø, A., Yokota, T., Hansen, C.N., Helge, J.W., Hey-Mogensen, M., and Dela, F. 2014. Two weeks of one-leg immobilization decreases skeletal muscle respiratory capacity equally in young and elderly men. Exp. Gerontol. 58: 269-278. doi:10.1016/j.exger.2014.08.013.

Halson, S.L. 2014. Sleep in Elite Athletes and Nutritional Interventions to Enhance Sleep. Sports Med. 44(S1): 13-23. doi:10.1007/s40279-014-0147-0.

Hansen, A.K., Fischer, C.P., Plomgaard, P., Andersen, J.L., Saltin, B., and Pedersen, B.K. 2005. Skeletal muscle adaptation: training twice every second day vs. training once daily. J. Appl. Physiol. 98(1): 93-99. doi:10.1152/japplphysiol.00163.2004.

Hawley, J.A., Burke, L.M., Phillips, S.M., and Spriet, L.L. 2011. Nutritional modulation of training-induced skeletal muscle adaptations. J. Appl. Physiol. 110(3): 834-845. doi:10.1152/japplphysiol.00949.2010.

Henriksson, J. 1977. Training induced adaptation of skeletal muscle and metabolism during submaximal exercise. J. Physiol. 270(3): 661-675.

Henriksson, J., and Reitman, J.S. 1977. Time course of changes in human skeletal muscle succinate dehydrogenase and cytochrome oxidase activities and maximal oxygen uptake with physical activity and inactivity. Acta Physiol. Scand. 99(1): 91-97. doi:10.1111/j.17481716.1977.tb10356.x.

Houston, M.E., Froese, E.A., Valeriote, S.P., Green, H.J., and Ranney, D.A. 1983. Muscle performance, morphology and metabolic capacity during strength training and detraining: a one leg model. Eur. J. Appl. Physiol. Occup. Physiol. 51(1): 25-35.

Janzen, C.L., Chilibeck, P.D., and Davison, K.S. 2006. The effect of unilateral and bilateral strength training on the bilateral deficit and lean tissue mass in post-menopausal women. Eur J. Appl. Physiol. 97(3): 253-260. doi:10.1007/s00421-006-0165-1.

Jensen, L., Bangsbo, J., and Hellsten, Y. 2004. Effect of high intensity training on capillarization and presence of angiogenic factors in human skeletal muscle. J. Physiol. 557(2): 571-582. doi:10.1113/jphysiol.2003.057711.

Kiens, B., Essen-Gustavsson, B., Christensen, N.J., and Saltin, B. 1993. Skeletal muscle 
substrate utilization during submaximal exercise in man: effect of endurance training. J. Physiol. 469: 459-478.

Kim, P.L., Staron, R.S., and Phillips, S.M. 2005. Fasted-state skeletal muscle protein synthesis after resistance exercise is altered with training. J. Physiol. 568(Pt 1): 283-290. doi:10.1113/jphysiol.2005.093708.

Klausen, K., Andersen, L.B., and Pelle, I. 1981. Adaptive changes in work capacity, skeletal muscle capillarization and enzyme levels during training and detraining. Acta Physiol. Scand. 113(1): 9-16. doi:10.1111/j.1748-1716.1981.tb06854.x.

Lamboley, C.R., Wyckelsma, V.L., Perry, B.D., McKenna, M.J., and Lamb, G.D. 2016. Effect of 23-day muscle disuse on sarcoplasmic reticulum $\mathrm{Ca} 2+$ properties and contractility in human type I and type II skeletal muscle fibers. J. Appl. Physiol. 121(2): 483-492. doi:10.1152/japplphysiol.00337.2016.

Lee, M., and Carroll, T.J. 2007. Cross education: possible mechanisms for the contralateral effects of unilateral resistance training. Sports Med. 37(1): 1-14.

Lexell, J., and Taylor, C.C. 1991. A morphometrical comparison of right and left whole human vastus lateralis muscle: how to reduce sampling errors in biopsy techniques. Clin. Physiol. 11(3): 271-276. doi:10.1111/j.1475-097X.1991.tb00458.x.

Lindholm, M.E., Giacomello, S., Werne Solnestam, B., Fischer, H., Huss, M., Kjellqvist, S., and Sundberg, C.J. 2016. The Impact of Endurance Training on Human Skeletal Muscle Memory, Global Isoform Expression and Novel Transcripts. PLoS Genet. 12(9): e1006294. doi:10.1371/journal.pgen.1006294.s018.

Lindholm, M.E., Huss, M., Solnestam, B.W., Kjellqvist, S., Lundeberg, J., and Sundberg, C.J. 2014. The human skeletal muscle transcriptome: sex differences, alternative splicing, and tissue homogeneity assessed with RNA sequencing. FASEB J. doi:10.1096/fj.14-255000.

Lundberg, T.R., Fernandez-Gonzalo, R., Gustafsson, T., and Tesch, P.A. 2013. Aerobic exercise does not compromise muscle hypertrophy response to short-term resistance training. J. Appl. Physiol. 114(1): 81-89. doi:10.1152/japplphysiol.01013.2012.

MacInnis, M.J., Zacharewicz, E., Martin, B.J., Haikalis, M.E., Skelly, L.E., Tarnopolsky, M.A., Murphy, R.M., and Gibala, M.J. 2016. Superior mitochondrial adaptations in human skeletal muscle after interval compared to continuous single-leg cycling matched for total work. J. Physiol. doi:10.1113/JP272570. 
Macnaughton, L.S., Wardle, S.L., Witard, O.C., McGlory, C., Hamilton, D.L., Jeromson, S., Lawrence, C.E., Wallis, G.A., and Tipton, K.D. 2016. The response of muscle protein synthesis following whole-body resistance exercise is greater following $40 \mathrm{~g}$ than $20 \mathrm{~g}$ of ingested whey protein. Physiol. Rep. 4(15). doi:10.14814/phy2.12893.

McCurdy, K.W., Langford, G.A., Doscher, M.W., Wiley, L.P., and Mallard, K.G. 2005. The effects of short-term unilateral and bilateral lower-body resistance training on measures of strength and power. J Strength Cond Res. 19(1): 9-15. doi:10.1519/14173.1.

McGlory, C., Wardle, S.L., Macnaughton, L.S., Witard, O.C., Scott, F., Dick, J., Bell, J.G., Phillips, S.M., Galloway, S.D.R., Hamilton, D.L., and Tipton, K.D. 2016. Fish oil supplementation suppresses resistance exercise and feeding $\square$ induced increases in anabolic signaling without affecting myofibrillar protein synthesis in young men. Physiol. Rep. 4(6): e12715. doi:10.14814/phy2.12715.

McPhee, J.S., Williams, A.G., Stewart, C., Baar, K., Schindler, J.P., Aldred, S., Maffulli, N., Sargeant, A.J., and Jones, D.A. 2009. The training stimulus experienced by the leg muscles during cycling in humans. Exp. Physiol. 94(6): 684-694. doi:10.1113/expphysiol.2008.045658.

Miller, B.F., Olesen, J.L., Hansen, M., Døssing, S., Crameri, R.M., Welling, R.J., Langberg, H., Flyvbjerg, A., Kjaer, M., Babraj, J.A., Smith, K., and Rennie, M.J. 2005. Coordinated collagen and muscle protein synthesis in human patella tendon and quadriceps muscle after exercise. J. Physiol. 567(3): 1021-1033. doi:10.1113/jphysiol.2005.093690.

Mitchell, C.J., Churchward-Venne, T.A., West, D.W.D., Burd, N.A., Breen, L., Baker, S.K., and Phillips, S.M. 2012. Resistance exercise load does not determine training-mediated hypertrophic gains in young men. J. Appl. Physiol. 113(1): 71-77. doi:10.1152/japplphysiol.00307.2012.

Mittendorfer, B., Andersen, J.L., Plomgaard, P., Saltin, B., Babraj, J.A., Smith, K., and Rennie, M.J. 2005. Protein synthesis rates in human muscles: neither anatomical location nor fibretype composition are major determinants. J. Physiol. 563(Pt 1): 203-211. doi:10.1113/jphysiol.2004.077180.

Morton, R.W., McGlory, C., and Phillips, S.M. 2015. Nutritional interventions to augment resistance training-induced skeletal muscle hypertrophy. Front. Physiol. 6: 525. doi:10.1016/j.bbi.2013.10.002. 
Morton, R.W., Oikawa, S.Y., Wavell, C.G., Mazara, N., McGlory, C., Quadrilatero, J., Baechler, B.L., Baker, S.K., and Phillips, S.M. 2016. Neither load nor systemic hormones determine resistance training-mediated hypertrophy or strength gains in resistance-trained young men. J. Appl. Physiol. 121(1): 129-138. doi:10.1152/japplphysiol.00154.2016.

Mourtzakis, M., Gonzalez-Alonso, J., Graham, T.E., and Saltin, B. 2004. Hemodynamics and O2 uptake during maximal knee extensor exercise in untrained and trained human quadriceps muscle: effects of hyperoxia. J. Appl. Physiol. 97(5): 1796-1802. doi:10.1152/japplphysiol.00169.2004.

Mujika, I., and Padilla, S. 2000. Detraining: loss of training-induced physiological and performance adaptations. Part I: short term insufficient training stimulus. Sports Med. 30(2): 79-87.

Munn, J. 2004. Contralateral effects of unilateral resistance training: a meta-analysis. J. Appl. Physiol. 96(5): 1861-1866. doi:10.1152/japplphysiol.00541.2003.

Newton, R.U., Gerber, A., Nimphius, S., Shim, J.K., Doan, B.K., Robertson, M., Pearson, D.R., Craig, B.W., Häkkinen, K., and Kraemer, W.J. 2006. Determination of Functional Strength Imbalance of the Lower Extremities. J. Strength Cond. Res. 20(4): 971. doi:10.1519/R$5050501 x .1$.

Perry, B.D., Wyckelsma, V.L., Murphy, R.M., Steward, C.H., Anderson, M., Levinger, I., Petersen, A.C., and McKenna, M.J. 2016. Dissociation between short-term unloading and resistance training effects on skeletal muscle $\mathrm{Na}+, \mathrm{K}+$-ATPase, muscle function, and fatigue in humans. J. Appl. Physiol. 121(5): 1074-1086. doi:10.1152/japplphysiol.00558.2016.

Rakobowchuk, M., Crozier, J., Glover, E.I., Yasuda, N., Phillips, S.M., Tarnopolsky, M.A., and Macdonald, M.J. 2011. Short-term unilateral leg immobilization alters peripheral but not central arterial structure and function in healthy young humans. Eur. J. Appl. Physiol. 111(2): 203-210. doi:10.1007/s00421-010-1636-y.

Rud, B., Foss, O., Krustrup, P., Secher, N.H., and Hallén, J. 2012. One-legged endurance training: leg blood flow and oxygen extraction during cycling exercise. Acta Physiol. (Oxf). 205(1): 177-185. doi:10.1111/j.1748-1716.2011.02383.x.

Safdar, A., Saleem, A., and Tarnopolsky, M.A. 2016. The potential of endurance exercisederived exosomes to treat metabolic diseases. Nat. Rev. Endocrinol. 12(9): 504-517. doi:10.1038/nrendo.2016.76. 
Saltin, B., Nazar, K., Costill, D.L., Stein, E., Jansson, E., Essén, B., and Gollnick, D. 1976. The nature of the training response; peripheral and central adaptations of one-legged exercise. Acta Physiol. Scand. 96(3): 289-305. doi:10.1111/j.1748-1716.1976.tb10200.x.

Schantz, P.G., Moritani, T., Karlson, E., Johansson, E., and Lundh, A. 1989. Maximal voluntary force of bilateral and unilateral leg extension. Acta Physiol. Scand. 136(2): 185-192. doi:10.1111/j.1748-1716.1989.tb08651.x.

Secher, N.H., Rube, N., and Elers, J. 1988. Strength of two- and one-leg extension in man. Acta Physiol. Scand. 134(3): 333-339. doi:10.1111/j.1748-1716.1988.tb08500.x.

Suetta, C., Hvid, L.G., Justesen, L., Christensen, U., Neergaard, K., Simonsen, L., Ortenblad, N., Magnusson, S.P., Kjaer, M., and Aagaard, P. 2009. Effects of aging on human skeletal muscle after immobilization and retraining. J. Appl. Physiol. 107(4): 1172-1180. doi:10.1152/japplphysiol.00290.2009.

Škarabot, J., Cronin, N., Strojnik, V., and Avela, J. 2016. Bilateral deficit in maximal force production. Eur. J. Appl. Physiol. doi:10.1007/s00421-016-3458-z.

Tarnopolsky, M., Phillips, S., Parise, G., Varbanov, A., Demuth, J., Stevens, P., Qu, A., Wang, F., and Isfort, R. 2007. Gene expression, fiber type, and strength are similar between left and right legs in older adults. J. Gerontol. A Biol. Sci. Med. Sci. 62(10): 1088-1095.

Tesch, P.A., Trieschman, J.T., Ekberg, A. 2004. Hypertrophy of chronically unloaded muscle subjected to resistance exercise. J. Appl. Physiol. 96(4): 1451-1458. doi:10.1152/japplphysiol.01051.2003.

Tesch, P.A., Lundberg, T.R., and Fernandez-Gonzalo, R. 2016. Unilateral lower limb suspension: From subject selection to “omic" responses. J. Appl. Physiol. 120(10): 12071214. doi:10.1152/japplphysiol.01052.2015.

Verdijk, L.B., Koopman, R., Schaart, G., Meijer, K., Savelberg, H.H.C.M., and van Loon, L.J.C. 2007. Satellite cell content is specifically reduced in type II skeletal muscle fibers in the elderly. Am. J. Physiol. Endocrinol. Metab. 292(1): E151-7. doi:10.1152/ajpendo.00278.2006.

Vigelsø, A., Gram, M., Wiuff, C., Andersen, J.L., Helge, J.W., and Dela, F. 2015a. Six weeks" aerobic retraining after two weeks" immobilization restores leg lean mass and aerobic capacity but does not fully rehabilitate leg strength in young and older men. J. Rehabil. Med. 47(6): 552-560. doi:10.2340/16501977-1961. 
Vigelsø, A., Gram, M., Wiuff, C., Hansen, C.N., Prats, C., Dela, F., and Helge, J.W. 2015 b. Effects of immobilization and aerobic training on proteins related to intramuscular substrate storage and metabolism in youngand older men. Eur. J. Appl. Physiol. 116(3): 481-494. doi:10.1007/s00421-015-3302-X.

Wall, B.T., Dirks, M.L., and van Loon, L.J.C. 2013. Ageing Res. Rev. 12(4): 898-906. doi:10.1016/j.arr.2013.07.003.

West, D.W.D., and Phillips, S.M. 2010. Anabolic processes in human skeletal muscle: restoring the identities of growth hormone and testosterone. Phys. Sportsmed 38(3): 97-104. doi:10.3810/psm.2010.10.1814.

West, D.W.D., and Phillips, S.M. 2012. Associations of exercise-induced hormone profiles and gains in strength and hypertrophy in a large cohort after weight training. Eur. J. Appl. Physiol. 112(7): 2693-2702. doi:10.1007/s00421-011-2246-Z.

West, D.W.D., Burd, N.A., Tang, J.E., Moore, D.R., Staples, A.W., Holwerda, A.M., Baker, S.K., and Phillips, S.M. 2010. Elevations in ostensibly anabolic hormones with resistance exercise enhance neither training-induced muscle hypertrophy nor strength of the elbow flexors. J. Appl. Physiol. 108(1): 60-67. doi:10.1152/japplphysiol.01147.2009.

West, D.W.D., Kujbida, G.W., Moore, D.R., Atherton, P., Burd, N.A., Padzik, J.P., De Lisio, M., Tang, J.E., Parise, G., Rennie, M.J., Baker, S.K., and Phillips, S.M. 2009. Resistance exercise-induced increases in putative anabolic hormones do not enhance muscle protein synthesis or intracellular signalling in young men. J. Physiol. 587(Pt 21): 5239-5247. doi:10.1113/jphysiol.2009.177220.

Whitham, M., and Febbraio, M.A. 2016. The ever-expanding myokinome: discovery challenges and therapeutic implications. Nat. Rev. Drug Discov. 15(10): 719-729. doi:10.1038/nrd.2016.153.

Wilkinson, S.B., Phillips, S.M., Atherton, P.J., Patel, R., Yarasheski, K.E., Tarnopolsky, M.A., and Rennie, M.J. 2008. Differential effects of resistance and endurance exercise in the fed state on signalling molecule phosphorylation and protein synthesis in human muscle. J. Physiol. 586(15): 3701-3717. doi:10.1113/jphysiol.2008.153916.

Wilkinson, S.B., Tarnopolsky, M.A., Grant, E.J., Correia, C.E., and Phillips, S.M. 2006. Hypertrophy with unilateral resistance exercise occurs without increases in endogenous anabolic hormone concentration. Eur. J. Appl. Physiol. 98(6): 546-555. doi:10.1007/s00421- 
006-0300-z.

Witard, O.C., Jackman, S.R., Breen, L., Smith, K., Selby, A., and Tipton, K.D. 2014.

Myofibrillar muscle protein synthesis rates subsequent to a meal in response to increasing doses of whey protein at rest and after resistance exercise. Am. J. Clin. Nutr. 99(1): 86-95. doi:10.3945/ajen.112.055517. 


\section{Figure legends}

Figure 1. A schematic of three different experimental designs used in studies of skeletal muscle remodeling in humans. In parallel group designs, different participants undergo each treatment, whereas in crossover designs, the same participants undergo each treatment, separated by a washout period (indicated by the arrow). In the first case, participants are randomized to groups, while for crossover designs participants are randomized to a sequence (A|B or $\mathrm{B} \mid \mathrm{A})$. With the unilateral exercise model, each leg is randomly assigned to a treatment, but each participant undergoes both treatments in close temporal succession. Note that for each of the above experimental designs, the same number of observations is collected, despite the greater number of participants needed for the parallel group design.

Figure 2. The effect of sample size on statistical power for between- and within-participant comparisons. The above plots were generated in $\mathrm{G}^{*}$ power v3.1.9.2 by performing student's ttests with sample sizes ranging from 10 to 200 , in increments of 10 . The mean differences ( $\bar{x}$ ) between the groups were 20 (red), 10 (green), and 5 (blue), with standard deviation of 20 for each group. The resulting effect sizes were $1,0.5$, and 0.25 for the between-participant comparisons (dashed lines) and 1.41, 0.71, and 0.35 (using a correlation coefficient of 0.75 ) for the within-participant comparisons (solid lines). For a given mean difference between groups, the threshold sample size required for $80 \%$ statistical power (dotted line) is lower for withinparticipant comparisons relative to between-participant comparisons.

Figure 3. Changes in physiological parameters of the non-immobilized limb before and after 14 days of unilateral leg immobilization. The data presented in panels A-F are from Glover et al. 2008. The control leg was unchanged with respect to (A) isometric maximal 
voluntary contraction (N.m), (B) thigh cross sectional area $\left(\mathrm{cm}^{2}\right),(\mathrm{C})$ type I muscle fibre cross sectional area $\left(\mu \mathrm{m}^{2}\right)$, (D) type II muscle fibre cross sectional area $\left(\mu \mathrm{m}^{2}\right)$, (E) citrate synthase activity ( $\mu \mathrm{mol} / \mathrm{kg} /$ protein $/ \mathrm{h})$, and (F) beta hydroxybutyrate activity ( $\mu \mathrm{mol} / \mathrm{kg} / \mathrm{protein} / \mathrm{h}$ ). 
Treatment A

Treatment B

Parallel-group $(n=20)$

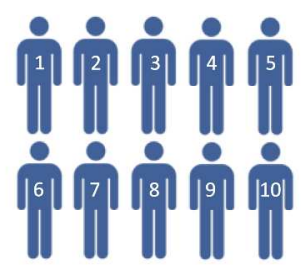

vs.

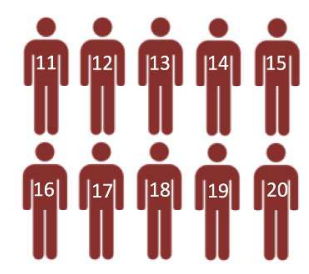

Crossover $(n=10)$

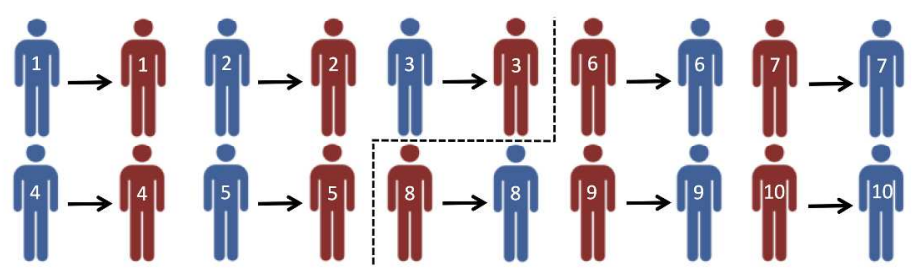

Unilateral exercise model $(n=10)$

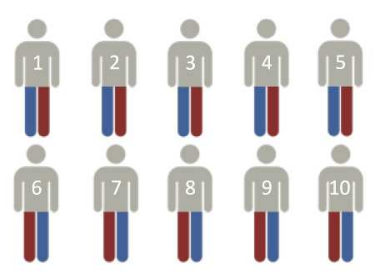

Figure 1 $385 \times 331 \mathrm{~mm}(300 \times 300$ DPI $)$ 


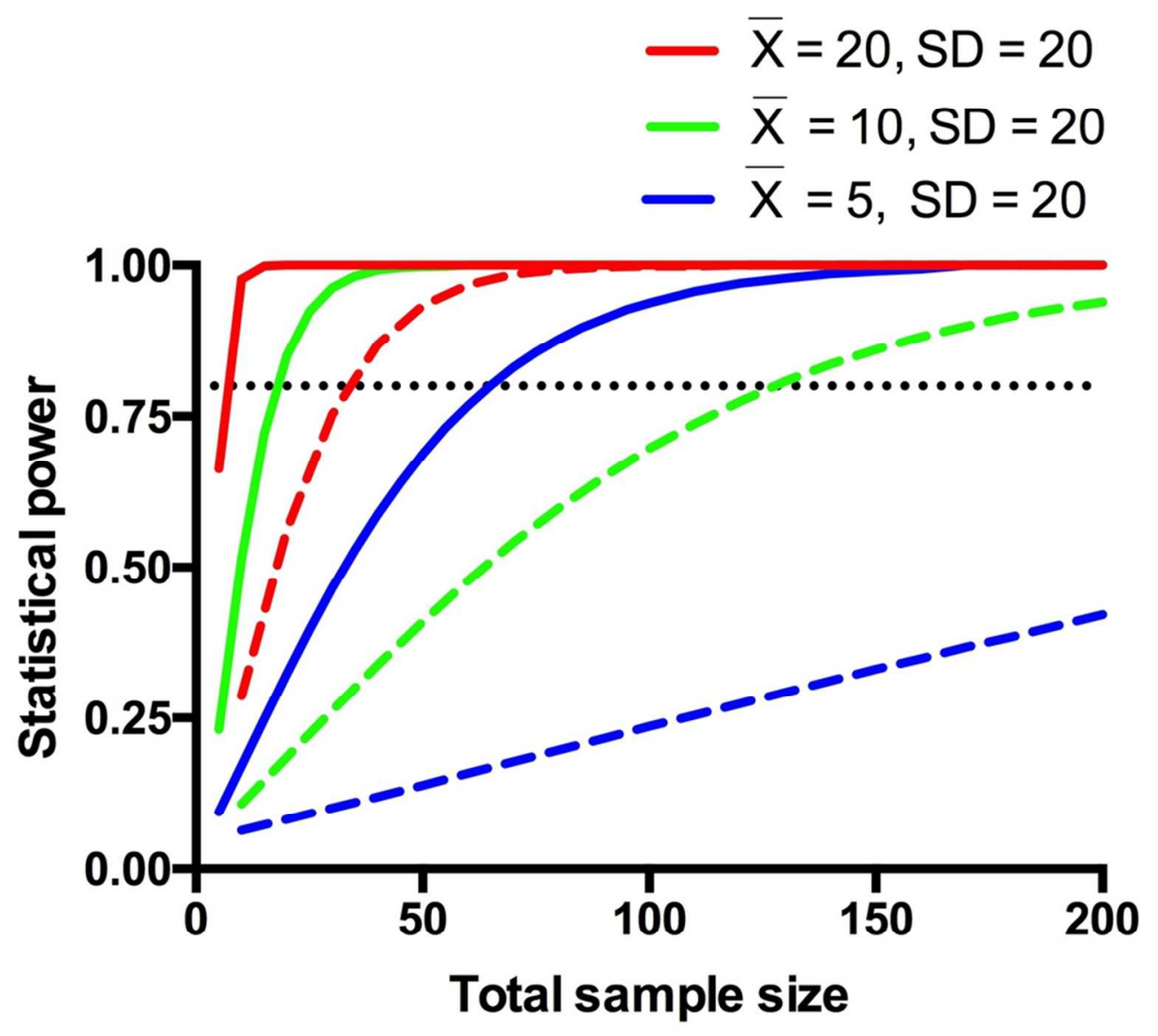

Figure 2

$88 \times 81 \mathrm{~mm}(300 \times 300$ DPI $)$ 
Page431 of 31

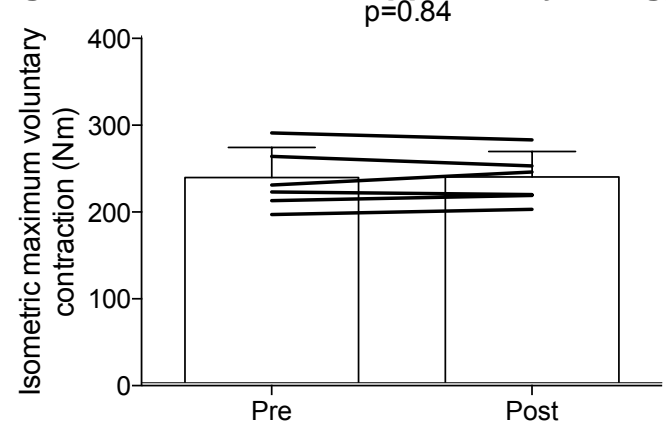

C

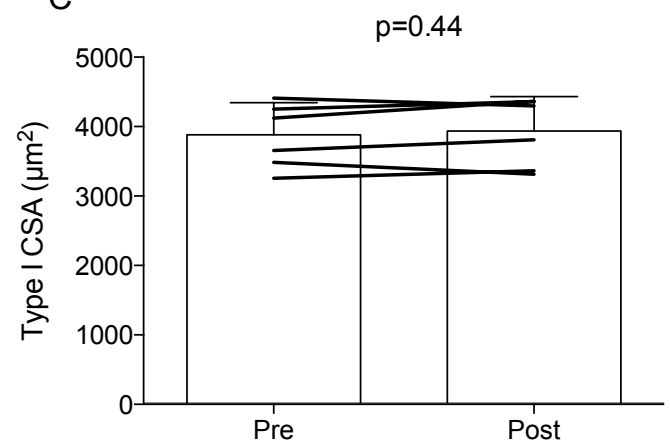

E

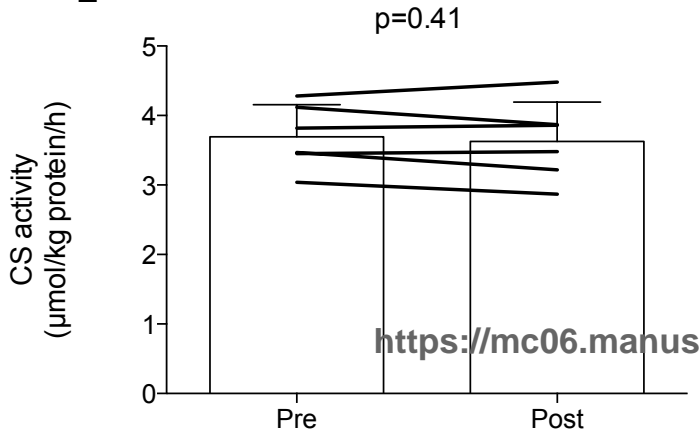

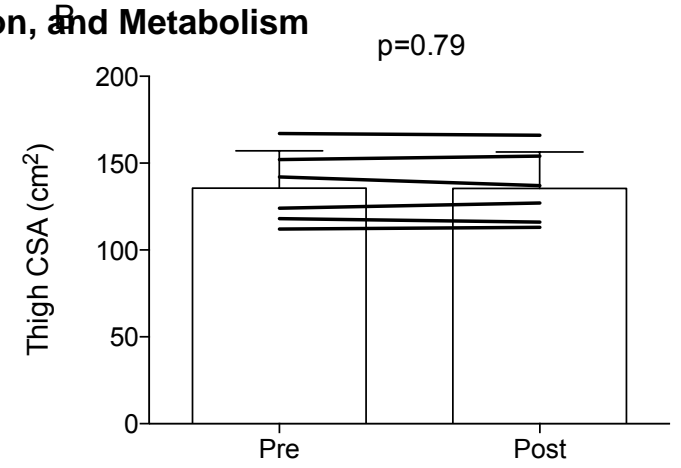

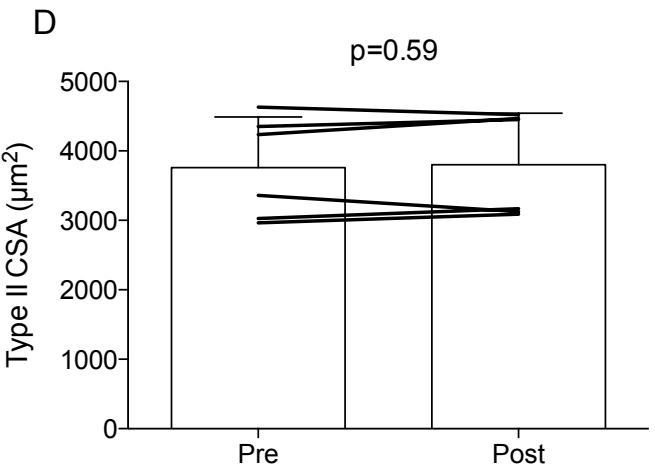

F

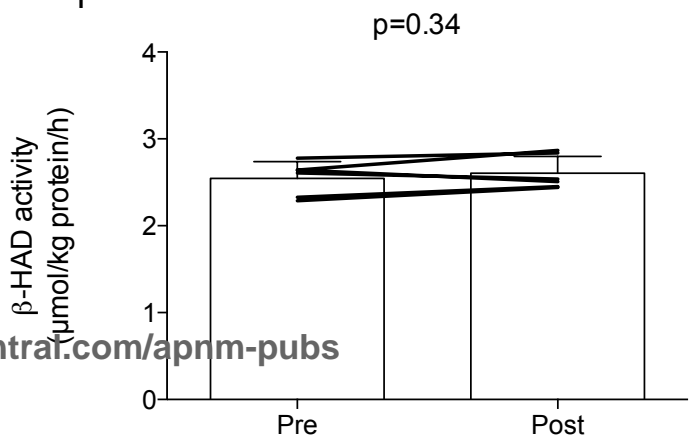

